\title{
Expression of cytoplasmic and nuclear Survivin in primary and secondary human glioblastoma
}

\author{
D Xie*,', YX Zeng', HJ Wang', JM Wen ${ }^{3}$, Y Tao ${ }^{3}$, JST Sham ${ }^{4}$ and XY Guan*,4 \\ 'State Key Laboratory of Oncology in Southern China, Cancer Center, Sun Yat-Sen University, Guangzhou, China; ${ }^{2}$ Department of Neurosurgery, the First \\ Affiliated Hospital, Sun Yat-Sen University, Guangzhou, China; ${ }^{3}$ Department of Pathology, Zhong Shan Medical College, Sun Yat-Sen University, \\ Guangzhou, China; ${ }^{4}$ Department of Clinical Oncology, University of Hong Kong, Hong Kong, China
}

Clinically, human glioblastoma (GBM) may develop de novo or from a low-grade glioma (secondary GBM), and molecular alterations in the two pathways may differ. This study examined the status of Survivin expression and apoptosis in 30 primary and 26 secondary GBMs. Our results show that cytoplasmic Survivin positivity was significantly $(P<0.001)$ more frequent in primary GBMs $(83 \%)$ than that in secondary GBMs (46\%). In addition, an inverse correlation of cytoplasmc Survivin positivity with GBM apoptotic index, and a positive association between cytoplasmic Survivin and size of the tumours were observed. These results suggest that cytoplasmic Survivin, via its antiapoptotic function, may be involved in the tumorigenesis of many primary GBMs, but only in a small fraction of secondary GBMs. Furthermore, the overall progression times from low-grade precursor lesions to secondary GBMs were significantly shorter $(P<0.05)$ in cytoplasmic Survivin-positive cases (mean, 15.6 months) than those in Survivin-negative cases (mean, 23.8 moths), and the positive expression level of Survivin in cytoplasm was upregulated in most secondary GBMs when compared to matched pre-existing low-graded lesions. These results suggest that the increased accumulation of Survivin in the cytoplasm of more malignant glioma cells may prove to be a selective advantage, thus accelerating progression to a more aggressive phenotype. British Journal of Cancer (2006) 94, I08-1 |4. doi:I0.1038/sj.bjc.6602904 www.bjcancer.com

Published online 13 December 2005

(c) 2006 Cancer Research UK

Keywords: glioblastoma multiform; Survivin; immunohistochemistry; apoptosis

Survivin is located on chromosome 17q25. It was initially identified as a gene with structural homology to a family of genes known as inhibitors of apoptosis (IAPs) (Ambrosini et al, 1997). Survivin is known to be normally expressed at high levels in fetal tissue but absent in most normal adult differentiated cells (Adida et al, 1998). It is expressed extensively in common human cancers, such as colorectal carcinoma (Kawasaki et al, 1998), lung cancer (Monzo et al, 1999), and oesophageal carcinoma (Kato et al, 2001), and the increased expression of Survivin appears to be associated with the aggressive nature and/or poor prognosis of the above cancers. It is thought that Survivin enhances survival of tumour cells primarily through its antiapoptotic function via direct blocking of the terminal effecter cell-death proteases, caspases-3 and -7 , which are distinct from the BCL-2 family of antiapoptotic proteins (Shin et al, 2001). In human gliomas, some reports have documented that the increased expression of cytoplasmic Survivin, detected by immunohistochemistry (IHC), in gliomas was correlated with an ascending pathological grade of tumour (Sasaki

* Correspondence: Dr D Xie, State Key Laboratory of Oncology in Southern China, Cancer Center, Sun Yat-Sen University, 65I Dongfeng Road East, Guangzhou, china; E-mail: danxie@graduate.hku.hk or Dr XY Guan, Department of Clinical Oncology, University of Hong Kong, Room 109, School of Chinese Medicine Building, 10 Sassoon Road, Hong Kong, China; E-mail: xyguan@hkucc.hku.hk

Received 10 June 2005; revised 27 October 2005; accepted 15 November 2005; published online 13 December 2005 et al, 2002; Jiao et al, 2004). In addition, in glioblastoma multiform (GBM, WHO, grade IV), patients with detectable Survivin expression by Western blot analysis have been observed to have significantly shorter overall survival times compared with those without detectable expression (Chakravarti et al, 2002). These results suggest that Survivin might be involved in tumorigenesis and progression of GBM.

It has been suggested that the development of GBM could be along two distinct pathogenic pathways (Watanabe et al, 1996). Primary GBM may arise from de novo after a short clinical history without an identifiable less-malignant precursor lesion and frequently contains EGFR amplification/overexpression, PTEN mutation, homozygous p16 deletion, and loss of heterozygosity (LOH) on chromosome 19q. Secondary GBM often develops more slowly from a low-grade or anaplastic astrocytoma. P53 mutations and $\mathrm{LOH}$ on chromosome $10 \mathrm{q}$ are often observed, while amplification/overexpression of EGFR is most often lacking (Watanabe et al, 1996; Nakamura et al, 2000). This suggests that primary and secondary GBMs develop differently on a genetic level. However, whether or not the expression of Survivin is different between primary and secondary GBMs and the potential role of Survivin in tumorigenesis of GBMs in different pathogenic pathways have not been described to date.

In the present study, we evaluated the protein expression of Survivin by IHC in a total of 56 GBMs, including 30 primary and 26 secondary GBMs, and in 15 matched low-grade precursor lesions (grade II or III gliomas) of secondary GBMs. Since Survivin has both nuclear and cytoplasmic targets, which appear to serve 
different functions (Mahotka et al, 2002), two different commercially available Survivin antibodies, which recognise predominantly nuclear and cytoplasmic forms of Survivin, were used (Altura et al, 2003). Nuclear Survivin has been suggested to play an important role in chromosomal segregation during mitosis (Uren et al, 2000; Honda et al, 2003), while cytoplasmic Survivin has been characterised as antiapoptotic (O'Connor et al, 2002). In addition, the TUNEL assay was used to detect the apoptotic status of the GBMs and apoptotic status was correlated with the expression of different Survivin forms. Our results, which extend previous results of others (Chakravarti et al, 2002; Sasaki et al, 2002; Jiao et al, 2004) that examined Survivin expression in human gliomas, are intended to shed light on our understanding of the developmental genetic mechanisms of primary and secondary GBMs.

\section{MATERIALS AND METHODS}

\section{Patients and tissues specimens}

In this study, 56 cases of GBM patients, whose clinical and pathological information were available in detail, were selected from the First Affiliated Hospital of Sun Yat-Sen University (SYSU, Guang Zhou, China) from January 1990 to January 2000. The age of patients at the time of surgery ranged from 9 to 67 years, and the male/female ratio was $2.1: 1$. In all, 45 tumours were localised in the hemispheres, six tumours were in the basal ganglia, and five tumours were distributed in the brain stem. A total of 35 GBMs were larger than $5 \mathrm{~cm}$ in diameter, while $21 \mathrm{GBMs}$ were no more than $5 \mathrm{~cm}$ in diameter.

Of the 56 patients studied, 30 were primary GBMs, 26 were secondary GBMs. Diagnostic criteria for primary and secondary GBM were according to a previous report (Watanabe et al, 1996). Primary GBM was defined on the basis of clinical history of less than 3 months and patient's pathological diagnosis at the first onset of GBM. The diagnosis of secondary GBM required at least two biopsies and clinical as well as histological evidence of progression from low-graded (II-III) glioma, but it was pathologically diagnosed as GBM at the last operation. The clinical history of secondary GBM was at minimum longer than 3 months. The biopsy specimens, processed into paraffin blocks, of both primary and secondary GBMs were obtained from the Department of Pathology and Neurological Surgery, the First Affiliated Hospital of SYSU. In addition, in these GBM series, 15 matched precursor lesions (low-graded glioma) for secondary GBMs were also available, in which 10 cases were grade II glioma and five were anaplastic glioma (grade III).

\section{Immunohistochemistry}

Immunohistochemistry studies were performed using the SABC method as previously described (Xie et al, 2003) with slight modifications. For antigen retrieval, slides were microwave-treated in a $50 \mathrm{~mm}$ citrate buffer and boiled $(\mathrm{pH} 6.0)$ for $10 \mathrm{~min}$. Nonspecific binding was blocked with $10 \%$ normal rabbit serum for $20 \mathrm{~min}$. The tumour slides were incubated at $4{ }^{\circ} \mathrm{C}$ overnight in a moist chamber with one of the two polyclonal anti-Survivin antibodies (NB-500-201 K3, Novus Biologicals, Littleton, CO, USA, $1: 300$ dilution and SC-10811, Santa Cruz Biotechnology, Santa Cruz, CA, USA, 1:400 dilution). Each antibody was known to detect predominantly cytoplasmic (NB-500-201 K3) or the nuclear (SC-10811) forms of Survivin (Altura et al, 2003). Negative controls were established by replacing the primary antibody with a normal rabbit Ig.

\section{Evaluation of IHC results}

Each IHC slide was assessed in 10 high-powered fields at $\times 40$ magnification, and Survivin staining was evaluated semiquantita- tively. A staining score (to ++++ ) was obtained as the proportion of immunopositive cells in each tumour. Expression of Survivin was scored as '-' for negative expression, '+' for positive staining in $<25$ cells, ' ++ ' for positive staining in $25-50 \%$ cells, ' +++ ' for positive staining in $51-75 \%$ cells, and ' ++++ ' for positive staining in $>75 \%$ cells.

\section{TUNEL assay}

The fluorescent TUNEL staining was performed using a Death Detection kit (Roche Diagnostic GmbH, Mannheim, Germany) according to the manufacturer's instructions. Briefly, the rehydrated section was microwave-treated in $10 \mathrm{~mm}$ citrate buffer ( $\mathrm{pH}$ 6.0) for $5 \mathrm{~min}$. After washing in PBS, the specimen was incubated with a mixture of TdT solution (enzyme solution) and FITC-lablled dUTP solution (fluorescence labled solution) in a humidified chamber in the dark at $37^{\circ} \mathrm{C}$ for $60 \mathrm{~min}$. After washing, the slide was examined with a Zeiss Axiophot fluorescence microscope. Negative controls were obtained by replacing the TdT solution with distilled water. The presence of clear nuclear staining (TUNEL positive, green colour) was indicative of apoptotic cells (Figure 1G). At least 1000 tumour cell nuclei were examined. The number of TUNEL-positive tumour cell nuclei was counted and the apoptotic index (AI) was determined as the percentage of apoptotic cells in the tumour. For evaluation of the TUNEL staining, the mean value of AI of all samples under study was often used as a cutoff value (Yamasaki et al, 1997). In this study, the mean value of AI for all GBM samples was 0.76 , thus, tumours were classified into two groups according to their AI: low AI group $(\mathrm{AI}<0.76)$ and high $\mathrm{AI}$ group $(\mathrm{AI} \geqslant 0.76)$.

\section{Statistical analysis}

The differences in Survivin expression between primary and secondary GBMs and the correlation of Survivin in GBM with patient's clinicopathological parameters and cell apoptosis were assessed by $\chi^{2}$ test. Unpaired $t$-test was used to assess the statistical significance of the differential recurrent times from precursor lesion to secondary GBM, between groups with and without Survivin expression. $P$-values of $<0.05$ were considered to be significant.

\section{RESULTS}

\section{Survivin expression in primary and secondary GBMs}

Using two different Survivin antibodies, we were able to detect predominantly cytoplasmic (NB-500-201 K3, Figure 1B-D) and nuclear (SC-10811, Figure 1A, E, and F) forms of Survivin in GBMs by IHC, although both antibodies had some overlapping reactivity. The results of Survivin expression in GBMs are outlined in Tables 1 and 2 . Of the 56 GBMs, $37(66 \%)$ had cytoplasmic positive staining of Survivin and $43(77 \%)$ had nuclear positive staining of Survivin. For the expression of Survivin in primary and secondary GBMs, the frequency of cytoplasmic positive staining of Survivin detected in primary GBMs was $83 \%$ (25 out of 30 ), which was significantly greater than that in secondary GBMs $(46 \%, 12$ out of 26) $(P<0.001)$. In contrast, nuclear expression of Survivin showed no significant difference between primary and secondary GBMs $(P=0.51)$, that is, the rate of nuclear positive staining of Survivin in the primary GBMs was $73 \%$ (22 out of 30 ), and in the secondary GBM group, $81 \%$ (21 out of 26 ).

\section{Survivin expression in secondary GBMs and matched precursor lesions}

Owing to the suggestion that increased expression of Survivin in human gliomas is positively correlated with an ascending 

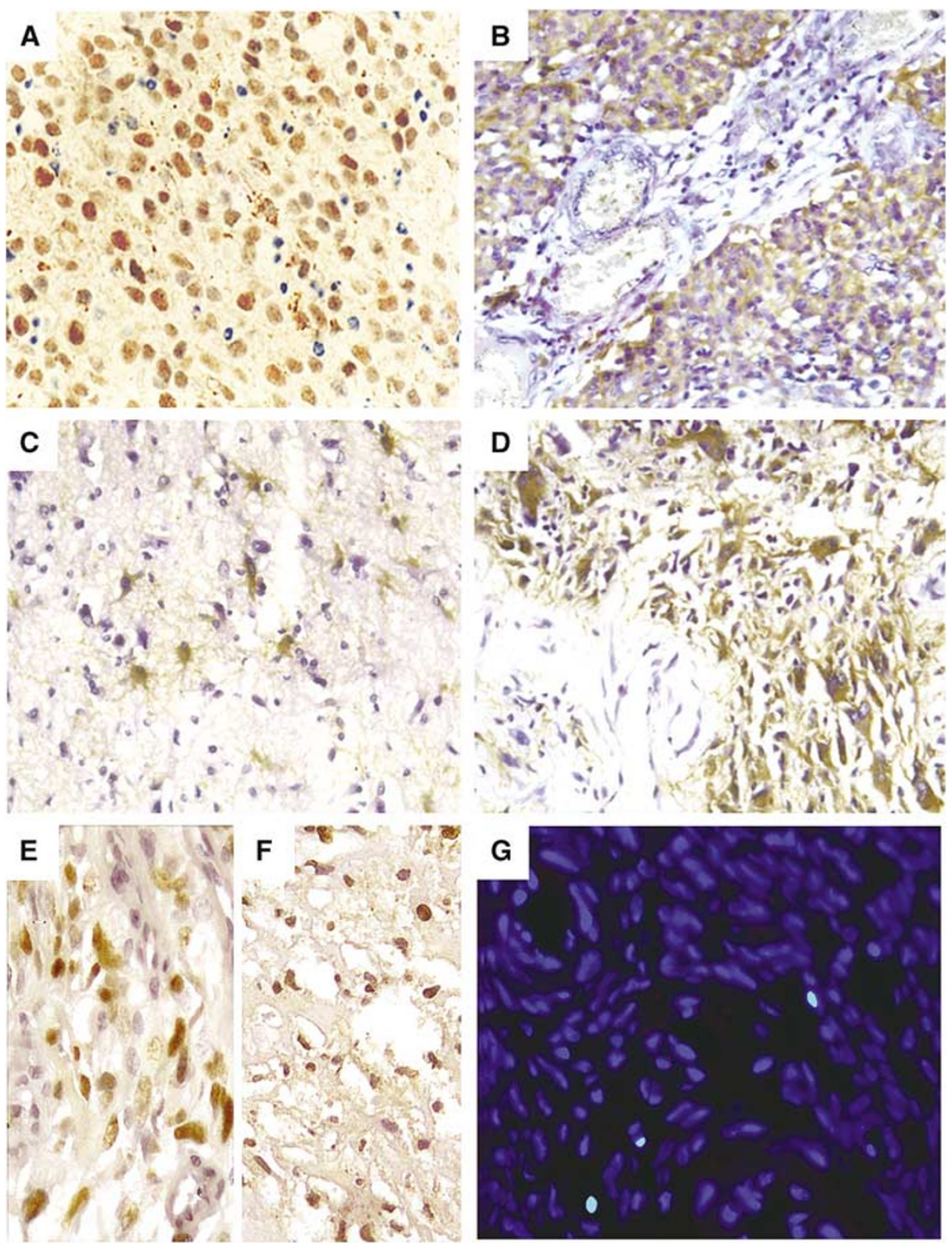

Figure I Immunohistochemical staining of Survivin and fluorescent TUNEL staining in human gliomas (A-G). A primary glioblastoma showed a high level $(++++)$ expression of nuclear Survivin $(\mathbf{A})$. High staining score $(++++)$ of cytoplasmic Survivin was detected in another primary glioblastoma $(\mathbf{B})$ Moderate expression level $(++)$ of cytoplasmic Survivin was detected in a pre-existing low-graded (grade II) glioma (C), which was obviously lower than that in its paired secondary glioblastoma with a staining score of $++++(\mathbf{D})$. High-level $(++++)$ expression of nuclear Survivin was detected in a secondary glioblastoma $(\mathbf{E})$ and its matched pre-existing grade II glioma $(\mathbf{F})$. Three apoptotic cells with clear nuclear staining (green color) were examined in a primary glioblastoma $(\mathbf{G})$.

pathological grade of tumour (Sasaki et al, 2002), we further evaluated the expression pattern of Survivin between 15 pairs of secondary GBMs and their pre-existing lower grade lesions. Overall, the expression level (staining score) of nuclear and cytoplasmic Survivin between the 15 matched secondary GBMs and precursor lesions was concordant. That is, of the seven secondary GBMs showing cytoplasmic positive of Survivin, six paired precursor lesions also had positive cytoplasmic Survivin staining and of the 11 secondary GBMs showing nuclear Survivin positive staining, each of the paired precursor lesions also showed positive nuclear Survivin (Table 3). The expression level (staining score) of cytoplasmic Survivin in the secondary GBMs was higher than that in paired pre-existing lower grade lesions in five of the seven paired cases (Table 3, Figure 1C and D). A difference in the expression level of nuclear Survivin between secondary GBMs and paired pre-existing lower grade lesions was not observed (Figure 1E and F).

\section{Association of Survivin expression with clinicopathological features}

A potential association between GBM Survivin expression and several known clinicopathological features, including patient's age, gender, tumour site, and tumour size, was further examined. No significant association was observed between Survivin nuclear expression and any of the patient's clinicopathological parameters $(P>0.05)$. In contrast, a significant association of Survivin cytoplasmic expression with tumour size was observed $(P<0.01)$. Thus, $80 \%$ ( 28 out of 35 ) of the large-sized GBMs (tumour size $\geqslant 5 \mathrm{~cm}$ in diameter) were found to have positive expression of 
Table I Status of Survivin expression and apoptotic index in primary GBMs

\begin{tabular}{|c|c|c|c|c|c|}
\hline $\begin{array}{l}\text { Case } \\
\text { no. }\end{array}$ & Age/sex & $\begin{array}{c}\text { Tumour } \\
\text { size }(\mathrm{cm})\end{array}$ & $\begin{array}{l}\text { Cytoplasmic } \\
\text { Survivin }^{\text {a }}\end{array}$ & $\begin{array}{l}\text { Nuclear } \\
\text { Survivin }^{\mathrm{a}}\end{array}$ & $A \mathbf{l}^{\mathbf{b}}$ \\
\hline I & ||$/ M$ & 5.7 & +++ & - & Low \\
\hline 2 & $15 / M$ & 3.1 & - & ++ & High \\
\hline 3 & $16 / F$ & 6.3 & ++++ & +++ & Low \\
\hline 4 & $16 / M$ & 7.8 & +++ & +++ & Low \\
\hline 5 & $19 / M$ & 4.3 & ++ & + & Low \\
\hline 6 & $23 / M$ & 5.6 & ++++ & ++++ & Low \\
\hline 7 & $27 / F$ & 7.3 & ++++ & +++ & High \\
\hline 8 & $28 / M$ & 8.5 & ++++ & ++++ & Low \\
\hline 9 & $30 / F$ & 6.4 & - & - & High \\
\hline 10 & $33 / M$ & 5.1 & +++ & ++ & High \\
\hline | | & $35 / M$ & 3.5 & ++++ & - & Low \\
\hline 12 & $35 / F$ & 5.2 & - & ++++ & High \\
\hline 13 & $36 / M$ & 7.5 & ++++ & ++++ & Low \\
\hline 14 & $37 / M$ & 10.2 & +++ & ++ & High \\
\hline 15 & $38 / M$ & 5.8 & +++ & - & Low \\
\hline 16 & $39 / M$ & 7.6 & ++++ & +++ & High \\
\hline 17 & $39 / M$ & 5.5 & + & +++ & Low \\
\hline 18 & $40 / M$ & 5.9 & +++ & - & Low \\
\hline 19 & $40 / M$ & 2.7 & - & + & High \\
\hline 20 & $4 I / M$ & 5.8 & + & - & Low \\
\hline 21 & $4 I / M$ & 3.4 & +++ & ++++ & Low \\
\hline 22 & $42 / F$ & 5.8 & ++++ & ++++ & Low \\
\hline 23 & $43 / M$ & 4.1 & ++++ & +++ & Low \\
\hline 24 & $45 / M$ & 11.3 & +++ & +++ & Low \\
\hline 25 & $46 / M$ & 4.4 & ++++ & ++ & High \\
\hline 26 & $49 / M$ & 9.2 & ++ & ++++ & Low \\
\hline 27 & $52 / F$ & 4.8 & - & + & High \\
\hline 28 & $53 / M$ & 5 & ++ & ++++ & Low \\
\hline 29 & $61 / M$ & 6.8 & ++++ & - & Low \\
\hline 30 & $67 / F$ & 12 & +++ & - & Low \\
\hline
\end{tabular}

aExpression of Survivin detected by immunohistochemistry were scored as '-' for negative expression, '+' for positivity in $<25$ cells, ' ++ ' for positivity in $25-50 \%$ cells, ' +++ ' for positivity in $5 \mathrm{I}-75 \%$ cells, and ' ++++ ' for positivity in $>75 \%$ cells. ${ }^{\mathrm{b}} \mathrm{Al}=$ apoptotic index; $\mathrm{Al} \geqslant 0.76$ were scored as 'high', $\mathrm{Al}<0.76$ were scored as 'low'.

cytoplasmic Survivin, while only $43 \%$ (nine out of 21 ) of the smallsized GBMs $(<5 \mathrm{~cm}$ in diameter) had positive expression of cytoplasmic Survivin. Cytoplasmic Survivin expression did not show a significant association with any other clinicopathological parameters of the patient $(P>0.05)$. In secondary GBMs, the mean progression times in months from precursor lesion onset to secondary GBMs was significantly shorter in cytoplasmic Survivinpositive cases (mean, 15.6 months) than that in cytoplasmic Survivin-negative cases (mean, 23.8 moths, $P<0.05$ ), (Table 2).

\section{Correlation of Survivin expression with cell apoptosis}

In this study, the TUNEL assay was used to study the status of apoptosis in all 56 GBMs. High AI $(\mathrm{AI} \geqslant 0.76)$ was detected in 23 out of $56(41 \%)$ of the tumours. Furthormore, a significant inverse correlation between Survivin cytoplasmic expression and tumour apoptosis was observed $(P<0.001)$, that is, in the GBMs with positive expression of cytoplasmic Survivin, $84 \%$ (31 out of 37) were observed to have a low AI $(\mathrm{AI}<0.76)$, whereas in the GBMs showing negativity of cytoplasmic Survivin (17 out of $19,89 \%$ ), a high AI $(A I \geqslant 0.76)$ was found (see Table 1 and 2). No significant correlation was observed between nuclear Survivin expression and GBM AI.

\section{DISCUSSION}

GBM, the most malignant of human brain glial tumours, may develop de novo (primary GBM) or via another pathogenic pathway, that is, from a low-grade glioma (secondary GBM) and the molecular alterations leading to the development of GBMs may differ (Nakamura et al, 2000). A previous report has documented a high frequent positive expression of Survivin in primary GBMs (Das et al, 2002). However, the expression pattern of this protein in secondary GBMs has not been described. In this study, the differences of Survivin expression between primary and secondary GBMs were examined by IHC using two different antibodies, one which detects predominantly cytoplasmic Survivin and one which detects predominantly nuclear forms of Survivin. Our results provide evidence that the expression pattern of nuclear Survivin was similar between primary and secondary GBMs, but, in contrast, cytoplasmic Survivin positivity was significantly more frequent in primary GBMs than that in secondary GBMs. It appears, therefore, that cytoplasmic expression of Survivin may provide additional insight into the developmental genetic mechanisms of GBM in different pathogenic pathways.

As discussed in detail in several intracellular localisation studies of Survivin protein, at present, at least three different splice variants of Survivin (ie, Survivin, Survivin delta $\operatorname{Ex~3,~and~}$ Survivin-2B) have been identified and have different subcellular localisations. Survivin and Survivin 2B isoforms localise predominantly to the cytoplasm, whereas the delta $\mathrm{Ex} 3$ isoform is preferentially localised in the nucleus (Mahotka et al, 2002). Nuclear and cytoplasmic pools of Survivin have distinct roles. Cytoplasmic Survivin has been characterised as antiapoptotic (O'Connor et al, 2002), while nuclear Survivin has been proposed to serve in the maintenance of the integrity of the mitotic spindle (Uren et al, 2000). Germane to previous studies (Jiao et al, 2004), we found an inverse correlation between the cytoplasmic expression of Survivin and cell apoptosis in our GBM cohorts, thus suggesting an antiapoptotic function of the cytoplasmic form of Survivin in GBMs. In addition, we observed a significant association between Survivin cytoplasmic expression and GBM tumour size. The positive of cytoplasmic Survivin expression was found more frequently in large-sized GBMs (tumour size $\geqslant 5 \mathrm{~cm}$ in diameter) than that in small-sized GBMs, the latter in which high-level cell apoptosis was more common. These results suggest that the antiapoptotic activity of cytoplasmic Survivin may be responsible, at least in part, for the growth and aggressive behaviour of GBM tumour cells.

In secondary GBMs, we found that the positive expression level of cytoplasmic Survivin was increased in most of secondary GBMs when compared to their matched low-grade tumours from the same patient, although the frequency of cytoplasmic Survivin positivity was similar to that in paired pre-existing low-graded lesions. In addition, the overall time in months of low-grade precursor lesion progression to secondary GBM was substantially less in cytoplasmic Survivin-positive cases than that of Survivinnegative cases. These results suggest a potential tumorigenic role of an upregulated expression of cytoplasmic Survivin along the linear progression model of low-grade lesions to secondary GBM. This also implies the possibility that the increased accumulation of Survivin in cytoplasm opposes apoptotic death in the early lessmalignant glioma cells and thus may provide a selective advantage and acceleration of progression from a lower grade glioma into a higher grade more malignant phenotype. These observations, in addition, may shed light on explaining why many gliomas, even at their earliest stages, are so resistant to conventional apoptosisrelated chemotherapy and radiation. However, it also appears that cytoplasmic Survivin accumulation is probably just one of several mechanisms that glioma cells may use to evade apoptosis, as we observed that more than $50 \%$ of secondary GBMs and their preexisting low-grade gliomas failed to express cytoplasmic Survivin.

An intriguing question that evolved in our studies is why is the rate of cytoplasmic Survivin in secondary GBMs significantly lower than that in primary GBMs? Although the pathogenic pathways leading to the development of GBMs may differ, almost all GBMs 
Table 2 Status of Survivin expression and apoptotic index in secondary GBMs

Preexisting lesion

\begin{tabular}{|c|c|c|c|c|c|c|c|}
\hline Case no. & Age/sex & Tumour size $(\mathrm{cm})$ & Cytoplasmic Survivin ${ }^{a}$ & Nuclear Survivin ${ }^{a}$ & $\mathbf{A l}^{\mathbf{b}}$ & Grade & Progressing time $^{c}$ \\
\hline 1 & $9 / M$ & 6.3 & - & +++ & Low & $\|$ & 18 \\
\hline 2 & $15 / M$ & 2.2 & - & - & High & $\|$ & 36 \\
\hline 3 & $16 / M$ & 5.8 & ++++ & +++ & Low & $\|$ & 31 \\
\hline 5 & $17 / M$ & 8.8 & - & +++ & High & $\|$ & 33 \\
\hline 6 & $18 / \mathrm{F}$ & 6.5 & +++ & ++++ & Low & III & 4 \\
\hline 7 & $23 / F$ & 4.9 & - & ++++ & High & $\|$ & 48 \\
\hline 8 & $25 / M$ & 6.4 & - & - & High & $\|$ & 23 \\
\hline 11 & $33 / F$ & 2.9 & - & + & High & III & 27 \\
\hline 12 & $35 / M$ & 7.2 & ++++ & +++ & Low & $\|$ & 22 \\
\hline 13 & $38 / F$ & 6.3 & ++ & +++ & Low & $\|$ & 21 \\
\hline 14 & 39/M & 4.3 & - & ++ & High & III & 32 \\
\hline 15 & $4 \mid / M$ & 9.3 & ++++ & - & Low & III & 7 \\
\hline 16 & $43 / F$ & 5.8 & - & ++ & High & $\|$ & 24 \\
\hline 17 & $43 / M$ & 3.0 & - & ++ & High & $\|$ & 11 \\
\hline 22 & $47 / M$ & 6.1 & ++++ & +++ & Low & III & 6 \\
\hline 23 & $49 / F$ & 2.3 & - & + & High & $\|$ & 24 \\
\hline 24 & $55 / F$ & 9.7 & ++++ & ++++ & Low & III & 16 \\
\hline 25 & $60 / M$ & 3.8 & - & ++++ & High & $\|$ & 5 \\
\hline 26 & $61 / M$ & 4.7 & ++++ & ++ & Low & III & 11 \\
\hline
\end{tabular}

a'Expression of Survivin detected by immunohistochemistry were scored as '-' for negative expression, ' + ' for positivity in $<25$ cells, ' ++ ' for positivity in $25-50 \%$ cells, ' ++++ ' for positivity in $5 \mathrm{I}-75 \%$ cells, and ' ++++ ' for positivity in $>75 \%$ cells. ${ }^{b} \mathrm{Al}=$ apoptotic index; $\mathrm{Al} \geqslant 0.76$ were scored as 'high', Al $<0.76$ were scored as 'low'. 'The times (months) form the first onset of low-grade glioma progressing to secondary GBM.

Table 3 The expression of cytoplasmic and nuclear Survivin in 15 paired secondary GBMs and pre-existing lesions ${ }^{a}$

\begin{tabular}{|c|c|c|c|c|}
\hline \multirow[b]{2}{*}{$\begin{array}{l}\text { Case } \\
\text { no. }\end{array}$} & \multicolumn{2}{|c|}{ Cytoplasmic Survivin } & \multicolumn{2}{|c|}{ Nuclear Survivin } \\
\hline & $\begin{array}{l}\text { Secondary } \\
\text { GBM }\end{array}$ & $\begin{array}{l}\text { Pre-existing } \\
\text { lesion }\end{array}$ & $\begin{array}{l}\text { Secondary } \\
\text { GBM }\end{array}$ & $\begin{array}{l}\text { Pre-existing } \\
\text { lesion }\end{array}$ \\
\hline I & - & - & +++ & +++ \\
\hline 3 & ++++ & ++ & +++ & ++++ \\
\hline 4 & ++++ & + & ++ & ++ \\
\hline 5 & - & - & +++ & +++ \\
\hline 7 & - & - & ++++ & ++++ \\
\hline 8 & - & - & - & - \\
\hline 10 & +++ & - & - & - \\
\hline 11 & - & - & + & ++ \\
\hline 15 & ++++ & +++ & - & - \\
\hline 17 & - & - & ++ & ++ \\
\hline 19 & - & - & - & - \\
\hline 21 & +++ & +++ & +++ & +++ \\
\hline 22 & ++++ & ++ & +++ & ++ \\
\hline 25 & - & - & ++++ & ++++ \\
\hline 26 & - & - & ++ & ++ \\
\hline
\end{tabular}

aExpression of Survivin detected by immunohistochemistry were scored as '-' for negative expression, '+' for positivity in $<25$ cells, '++' for positivity in $25-50 \%$ cells, ' +++ ' for positivity in $5 \mathrm{I}-75 \%$ cells, and ' ++++ ' for positivity in $>75 \%$ cells.

tend to possess active proliferation, high invasiveness and angiogenesis potential, resistance to conventional cytotoxic treatments and most of these patients have survival times less than 1 year (Davis et al, 1999). These similar neuropathologic and behavioural features of both primary and secondary GBMs imply that the criteria of clinicopathological classification for GBM may not reflect their malignant potential. In addition, it is also true that some of the primary GBMs may have rapidly progressed from lower grade gliomas, which often are clinically asymptomatic. Indeed, the positive expression levels of cytoplasmic Survivin in our primary GBMs were similar to that in our secondary GBMs, although the frequency of positivity was substantially higher in the former than in the later. These observations, collectively, prompt us to hypothesise that the high frequency of cytoplasmic Survivin in primary GBM may increase the barriers to cell apoptosis, accelerate the oncogenic process of primary GBM, in particular, facilitate the development of primary GBM in a short period without an identifiable less-malignant pre-existing lesion. Thus, if the above hypothesis has validity, it is not so difficult to understand why Survivin accumulation in cytoplasm is much more frequent in primary GBMs than that in secondary GBMs.

For the nuclear form of Survivin, we found that its expression pattern in secondary GBMs and paired low-graded precursor lesions was almost concordant. This was consistent, in part, with other observations, where no differential expression of nuclear Survivin was observed between independent low-graded gliomas and GBMs (Kleinschmidt-DeMasters et al, 2003). This suggests the possibility that the positive expression of the nuclear form of Survivin may be an early molecular event involved in the development of secondary GBMs. Although the potential role of nuclear Survivin and its mechanism of action in human cancers are still unclear, Honda et al (2003) recently have observed that in the nucleus, Survivin interacted with INCENP and aurora B kinase; these enzymes have an important role in chromosomal segregation during mitosis. Moreover, knockout or inhibition of Survivin has been shown to result in multinucleated and polyploid cells, which 
is a characteristic of mitotic arrest (Speliotes et al, 2000). In our GBM studies, the majority of GBMs showed an aneuploid DNA content (Xie et al, 2005), and, furthermore, a close association between nuclear Survivin positivity and tumour aneuploidy was observed (data not shown). These results suggest that the nuclear form of Survivin in GBMs may influence mitotic events and subsequently facilitate chromosomal instability. It is known that genetic instability can cause cytogenetic heterogeneity within a number of tumour types, including gliomas (Harada et al, 1998). Thus, it is quite possible that the widespread chromosomal instability associated with nuclear expression of Survivin observed in many GBMs can determine certain GBM histopathological characteristics, such as the presence of tumour cell heterogeneity and/or multiform tumour cells. However, the prognostic significance of nuclear Survivin among human cancers does vary in different tumour types. Recently, nuclear Survivin positivity has been reported to be predictive of poor survival in patients with oesophageal carcinoma and non-small-cell lung cancer (Grabowski et al, 2003; Lu et al, 2004). In contrast, high nuclear Survivin expression has been shown to be an independent indicator of a favourable prognosis in osteosarcoma, breast cancer, and gastric carcinomas (Okada et al, 2001; Kennedy et al, 2003; Trieb et al, 2003) and, moreover, it has been associated with a less-progressive cytologic grade in pediatric ependymomas and choroid plexus tumours of the brain (Altura et al, 2003). As regards to human glioma, more recently, the index of nuclear expression of Survivin

\section{REFERENCES}

Adida C, Crotty P, McGrath J, Berrebi D, Diebold J, Altieri D (1998) Developmentally regulated expression of the novel cancer anti-apoptosis gene survivin in human and mouse differentiation. Am J Pathol 152: $43-49$

Altura RA, Olshefski RS, Jiang Y, Boue DR (2003) Nuclear expression of survivin in paediatric ependymomas and choroid plexus tumours correlates with morphologic tumour grade. Br J Cancer 89: 1743 - 1749

Ambrosini G, Adida C, Altieri DC (1997) A novel anti-apoptosis gene, survivin, expressed in cancer and lymphoma. Nat Med 3: 917-921

Chakravarti A, Noll E, Black PM, Finkelstein DF, Finkelstein DM, Dyson NJ, Loeffler JS (2002) Quantitatively determined survivin expression levels are of prognostic value in human gliomas. J Clin Oncol 20: 1063-1068

Das A, Tan WL, Teo J, Smith DR (2002) Expression of survivin in primary glioblastomas. J Cancer Res Clin Oncol 128: 302-306

Davis FG, McCarthy BJ, Freels S, Al E (1999) The conditional probability of survival of patients with primary malignant brain tumors: surveillance, epidemiology, and end results (SEER) data. Cancer 85: 485-491

Grabowski P, Kuhnel T, Muhr-Wilkenshoff F, Heine B, Stein H, Hopfner M, Germer CT, Scherubl H (2003) Prognostic value of nuclear survivin expression in oesophageal squamous cell carcinoma. $\mathrm{Br} \mathrm{J}$ Cancer 88: $115-119$

Harada K, Nishizaki T, Ozaki S, Kubota H, Ito H, Sasaki K (1998) Intratumoral cytogenetic heterogeneity detected by comparative genomic hybridization and laser scanning cytometry in human gliomas. Cancer Res 58: $4694-4700$

Honda R, Korner R, Nigg EA (2003) Exploring the functional interactions between Aurora B, INCENP, and survivin in mitosis. Mol Biol Cell 14: $3325-3341$

Jiao B, Yao Z, Geng S, Zuo S (2004) Expression of survivin, a novel apoptosis inhibitor and cell regulatory protein, in human gliomas. Chin Med J 117: 612-614

Kato J, Kuwabara Y, Mitani M, Shinoda N, Sato A, Toyama T, Mitsui A, Nishiwaki T, Moriyama S, Kudo J, Fujii Y (2001) Expression of survivin in esophageal cancer: correlation with the prognosis and response to chemotherapy. Int J Cancer 95: $92-95$

Kawasaki H, Altieri D, Lu C, Toyoda M, Tenjo T, Tanigawa N (1998) Inhibition of apoptosis by survivin predicts shorter survival rates in colorectal cancer. Cancer Res 58: $5071-5074$

Kennedy SM, O’Driscoll L, Purcell R, Fitz-Simons N, McDermott EW, Hill AD, O'Higgins NJ, Parkinson M, Linehan R, Clynes M (2003) Prognostic importance of survivin in breast cancer. Br J Cancer 88: 1077-1083 has been observed to have a strong reverse association with the overall survival time of glioma patients in different grades (Uematsu et al, 2005). However, no prognostic impact of nuclear expression of Survivin in GBM was observed (Preusser et al, 2005). These observations suggest that the action of nuclear Survivin in tumour cells may be tumour specific. Further studies are clearly needed to elucidate the underlying function of nuclear Survivin in GBMs as well as in other human cancers.

In summary, in the present study, we describe for the first time the expression pattern of both nuclear and cytoplamic forms of Survivin in primary and secondary GBMs. Our results indicate that these different forms of Survivin (nuclear and cytoplasmic) have different functions in influencing the malignant behaviour of human glioma cells and these would have a profound effect on the development and/or progression of primary and secondary GBMs. Further studies designed to determine whether or not there is an association between cytoplasmic and/or nuclear expression of Survivin in GBM and GBM patients' clinical outcomes are clearly in order.

\section{ACKNOWLEDGEMENTS}

This study was supported by the Foundation of Developmental Project of Science and Technology of Guang Dong, and Leung Kwok Tze Foundation of Hong Kong, China.
Kleinschmidt-DeMasters BK, Heinz D, McCarthy PJ, Bobak JB, Lillehei KO, Shroyer ALW, Shroyer KR (2003) Survivin in glioblastomas, protein and messenger RNA expression and comparison with telomerase level. Arch Pathol Lab Med 127: 826-833

Lu B, Gonzalez A, Massion PP, Shyr Y, Shaktour B, Carbone DP, Hallahan DE (2004) Nuclear survivin as a biomarker for non-small-cell lung cancer. Br J Cancer 91: 537-540

Mahotka C, Liebmann J, Wenzel M, Suschek CV, Schmitt M, Gabbert HE, Gerharz CD (2002) Differential subcellular localization of functionally divergent survivin splice variants. Cell Death Differ 9: 1334-1342

Monzo M, Rosell R, Felip E, Astudillo J, Sanchez J, Maestre J, Martin C, Font A, Barnadas A, Abad A (1999) A novel antiapoptosis gene: reexpression of survivin messenger RNA as a prognosis marker in nonsmall-cell lung cancers. J Clin Oncol 17: 2100-2104

Nakamura M, Yang F, Fujisawa H, Yonekawa Y, Kleihues P, Ohgaki H (2000) Loss of heterozygosity on chromosome 19 in secondary glioblastomas. J Neuropathol Exp Neurol 59: 539-543

O'Connor DS, Wall NR, Porter AC, Altieri DC (2002) A p34(cdc2) survival checkpoint in cancer. Cancer Cell 2: 43-54

Okada E, Murai Y, Matsui K, Isizawa S, Cheng C, Masuda M, Takano Y (2001) Survivin expression in tumor cell nuclei is predictive of a favorable prognosis in gastric cancer patients. Cancer Lett 163: 109-116

Preusser M, Gelpi E, Matej R, Marosi C, Dieckmann K, Rossler K, Budka H, Hainfellner JA (2005) No prognostic impact of survivin expression in glioblastoma. Acta Neuropathol 109: 534-538

Sasaki T, Lopes MB, Hankins GR, Helm GA (2002) Expression of survivin, an inhibitor of apoptosis protein, in tumors of the nervous system. Acta Neuropathol 104: 105-109

Shin S, Sung BJ, Cho YS, Kim HJ, Ha NC, Hwang JI, Chung CW, Jung YK, Oh BH (2001) An anti-apoptotic protein human survivin is a direct inhibitor of caspase-3 and -7. Biochemistry 40: 1117-1123

Speliotes EK, Uren A, Vaux D, Horvitz HR (2000) Survivin and the inner centromere protein INCENP show similar cell-cycle localization and gene knockout phenotype. Curr Biol 10: 1319-1328

Trieb K, Lehner R, Stulnig T, Sulzbacher I, Shroyer KR (2003) Survivin expression in human osteosarcoma is a marker for survival. Eur J Surg Oncol 29: $379-382$

Uematsu M, Ohsawa I, Aokage T, Nishimaki K, Matsumoto K, Takahashi H, Asoh S, Teramoto A, Ohta S (2005) Prognostic significance of the immunohistochemical index of survivin in glioma: a comparative study with the MIB-1 index. J Neurooncol 72: 231-238 
Uren AG, Wong L, Pakusch M, Fowler KJ, Burrows FJ, Vaux DL, Choo KH (2000) Survivin and the inner centromere protein INCENP show similar cell-cycle localization and gene knockout phenotype. Curr Biol 10: $1319-1328$

Watanabe K, Tachibana O, Sato K, Yonekawa Y, Kleihues P, Ohgaki H (1996) Overexpression of the EGF receptor and p53 mutations are mutually exclusive in the evolution of primary and secondary glioblastomas. Brain Pathol 6: 217-224

Xie D, Sham JS, Zeng WF, Lin HL, Che LH, Wu HX, Wen JM, Fang Y, $\mathrm{Hu}$ L, Guan XY (2003) Heterogeneous expression and association of

beta-catenin, p16 and c-myc in multistage colorectal tumorigenesis and progression detected by tissue microarray. Int J Cancer 107: 896-902

Xie D, Zeng YX, Wang HJ, Tai LS, Wen JM, Tao Y, Ma NF, Hu L, Sham JS, Guan XY (2005) Amplification and overexpression of epidermal growth factor receptor gene in glioblastomas of Chinese patients correlates with patient/s age but not with tumor's clinicopathological pathway. Acta Neuropathol [Epub ahead of print]

Yamasaki F, Tokunaga O, Sugimori H (1997) Apoptotic index in ovarian carcinoma: correlation with clinicopathologic factors and prognosis. Gynecol Oncol 66: 439-448 